\title{
ORT.07 - Development of Digital Image Processing Methodology WT-MO: An Algorithm of High Accuracy in Detection And Counting of Erythrocytes, Leucocytes, Blasts
}

Ana Carolina Borges Monteiro ${ }^{1}$; Yuzo Iano ${ }^{1}$; Reinaldo Padilha França ${ }^{1}$; Rangel Arthur ${ }^{2}$; Vânia Vieira Estrela ${ }^{3 *}$; Abel Dueñas Rodriguez ${ }^{1}$; Sergio Luiz de Lima Assumpção ${ }^{4}$.

1UNICAMP - Faculdade de Engenharia Elétrica e Computação (FEEC);

2UNICAMP - Faculdade de Tecnologia (FT);

3UFF - Universidade Federal Fluminense;

4Fiocruz/Bio-Manguinhos;

Introduction: Annually 257.000 people in the world are diagnosed with leukemia. Leukemia is classified as a disorder in the production of blood cells within the bone marrow, and can be triggered from genetic factors to external factors such as chemical agents and radiation. Its main laboratory finding is the presence of blasts in the bloodstream, which can be easily detected by a hemogram, and the early diagnosis is of great importance for the cure rate.

However, early diagnosis is often neglected due to the cost of the blood count. In developed countries like the USA, the state does not guarantee health coverage of the population, while in developing countries there is a long queue waiting for the performance of examinations, in underdeveloped countries there is a population that lives on less than a dollar a day, making access to health difficult and other basic needs.

Objective: In view of the foregoing, reducing the costs of the hemogram test without the loss of the reliability of the examination is a primordial necessity. The present study aims to develop an algorithm of segmentation of digital images that is able to detect red blood cells, leukocytes and blasts by means of blood smear images, replacing the use of high-cost hematological equipment by a simple computer.

Methodology: For this, an algorithm based on the image processing techniques, Watershed Transform and morphological operations was developed in Matlab (2014a) software, thus giving rise to the WT-MO methodology.

In this context, the Watershed Transform is responsible for creating sets of pixels that have aspects similar to the staining and size of a red blood cell. After detection, each red cell is labeled with a number according to the counting order. In turn, morphological operations are responsible for creating a mask over the original image and removing all morphologically resembling pixels with leukocytes, either in full maturation state or in blastoid morphology. Counts results were released separately.

Results: The experiments were conducted using 10 digital blood smears obtained from online and open access platforms. The mean number of red cells and leukocytes per field was 61 and 14 cells, respectively. The results showed high accuracy in the detection of red blood cells (90\%) and leukocytes (90\%), as well as sensitivity (90\%) and specificity (90\%).

Conclusion: The WT-MO algorithm was computer-tested with an Intel Corei3 processor to determine how many seconds the methodology requires to analyze an image. In this way, the average execution time was 3.32 seconds. Therefore, the WT-MO methodology can be seen as the first step to perform the hemogram test with low cost and without loss of quality, speed and reliability.

Keywords: WT-MO algorithm; Blood Smear; Digital Image Processing 